\title{
Why and how have drug discovery strategies in pharma changed? What are the new mindsets?
}

\author{
Serge Mignani ${ }^{1,}{ }^{,}$, Scot Huber ${ }^{2}$, Helena Tomás ${ }^{3}$, João Rodrigues ${ }^{3,}$, \\ and Jean-Pierre Majoral| ${ }^{4,5},{ }^{*}$
}

\author{
${ }^{1}$ Universitf Paris Descartes, PRES Sorbonne Paris Citf, CNRS UMR 860, Laboratoire de Chimie et de \\ Biochimie Pharmacologiques et Toxicologique, 45, rue des Saints Peres, 75006 Paris, France \\ ${ }^{2}$ SCYNEXIS Inc., P.O. Box 12878, Research Triangle Park, North Carolina 27709, USA \\ ${ }^{3} \mathrm{CQM}$ - Centro de Química da Madeira, MMRG, Universidade da Madeira, Campus Universitário da \\ Penteada, 9000-390 Funchal, Portugal \\ ${ }^{4}$ Laboratoire de Chimie de Coordination du CNRS, 205 route de Narbonne, BP 44099, 31077 Toulouse \\ Cedex 4, France \\ ${ }^{5}$ Université de Toulouse, UPS, INPT 31077 Toulouse Cedex 4, France
}

Teaser: The key points emerging from the different R\&D approaches and enabling all scientists working in the drug discovery domain to develop innovative medicines for the 21st century are provided in this original review.

Keywords: Open innovation; generics; biosimilar; pheontypic and target based discovery; best-in-class; first-in-class; personalized medicine; companion diagnostics.

*Corresponding authors: Mignani, S. (serge.mignani@parisdescartes.fr); Rodrigues, J. (joaor@uma.pt); Majoral, J-P. (majoral@Icc-toulouse.fr).

\section{Author biographies}

\section{Serge Mignani}

Serge Mignani joined the Catholic University of Louvain-la-Neuve (Belgium) for a PhD training under the supervision of Professor H.G. Viehe. Then, he joined the University of Madison (USA) for a postdoctoral stay under the supervision of Professor B.M. Trost. In 1981 he joined Rhône-Poulenc (currently Sanofi) at the Vitry Research Center, where he was Head of the Medicinal Chemistry Department and Scientific Director. More than ten clinical candidates have been disclosed by his team. Serge Mignani is author of more than 120 publications and over 100 patents. More than 70 invited plenary lectures and conferences have been presented. Serge Mignani was nominated (2014) as Professor at the EuroMediterranean University of Fez, Morocco.

\section{João Rodrigues}

João Rodrigues received his PhD (1999) in chemistry/inorganic chemistry from the University of Lisbon (Portugal). He is a staff member of the University of Madeira (Madeira Island, Portugal) and responsible for the areas of inorganic and organometallic chemistry, nanochemistry and nanomaterials. Since 2013, he has belonged to the General Council of the University of Madeira. Since 2006, he has been the head of Centro de Química da Madeira (National Research Unit) and since 2012 he has been the director of the Master in Nanochemistry and Nanomaterials. Presently, he belongs to the board responsible for the roadmap for health and wellness in the framework of 'Horizon 2020' (Madeira 14-20) at Madeira Island (Portugal). He was a visiting scientist in several universities abroad (namely in China, India and Brazil) and serves as a referee for several peer-reviewed journals. His students (Post-docs, PhDs and Masters) 
have obtained positions in several universities and companies in Portugal and abroad (e.g. USA, Sweden, Brazil and China). His main research interests are based on the design, synthesis and characterization of dendrimers/metallodendrimers, molecular wires, nanoparticles and polymers for optoelectronic and biomedical applications (e.g. emerging and infectious diseases and oncology).

\section{Jean-Pierre Majoral}

Jean-Pierre Majoral is Emeritus Director of Research at the CNRS in Toulouse. His research interest is focused on the design and the properties of macromolecules such as phosphorus dendrimers and hyperbranched polymers, with key efforts directed to the use of dendrimers in medicinal chemistry and material sciences. Emphasis is also on immobilization of molecular and macromolecular organo- and metalo-catalysts and their use for fine chemical synthesis. He has received numerous prestigious awards from Germany, Poland, Spain, UK and France. He is a member of several Academies of Sciences worldwide and an author of 590 publications and 45 patents. 
In the pharmaceutical industry the long-term challenge of drug innovation is the key phrase throughout R\&D that refers to increasing the output of original drug candidate molecules. To increase R\&D productivity, implementation of new and strategic R\&D orientations to develop new approaches or systems to identify hits and leads efficiently has taken place and enabled all scientists working in the drug discovery domain to develop innovative medicines for the 21st century. 


\section{Introduction}

The pharmaceutical industry is currently facing huge challenges to overcome strongly growing economic pressure. Pharmaceutical companies must find new approaches to improve productivity and increase their output of original drugs, not only to satisfy demands of the healthcare sector to increase the life expectancy and quality of life of billions of people but also to ensure their business survival in terms of return on R\&D investment [1,2]. Today, R\&D organizations must strive to achieve drug innovation to address important issues such as the strong decrease in the number of new chemical entities launched every year and the eroding patent estate. Their main objective is to deliver hits, leads and finally drug candidates of the highest possible quality to decrease the risk of failure in clinical phases at the end of the development process.

Herein, we highlight and analyze the key points emerging from the different R\&D approaches that have been implemented and that have offered scientists working in the drug discovery domain insight into the invention and development of innovative medicines of the 21st century. The question posed by everybody involved in drug discovery is which of these key points represent the best strategy to create and to develop new drugs? The aim of this work is to provide the readers with a broad vision for new hit, lead and drug optimization processes using the space concept strategy and metrics.

\section{The pharmaceutical industry's grand challenge}

\section{Economic impact of the pharmaceutical industry}

The economic impact of biopharmaceuticals can be simply evaluated by the number of direct jobs, which is more than 810000 , and of total jobs (including indirect and induced jobs), which is nearly 3.4 million, created by the sector [3]. The market could be worth nearly US\$1.6 trillion by 2020 , up from US\$1.1 trillion in 2011 [4]. The R\&D-based pharmaceutical industry spends over US\$100 billion per year [5]. Clinical trials account for $63 \%$ of this, whereas the cost of preclinical drug discovery and development is estimated to be only 32\% [6]. In 2011, 5408 medicines were in clinical development worldwide, with around 2000 each in Phase I and Phase II, and approximately 850 were in Phase III. Many of the medicines in clinical development are for more than one medical application [3].

\section{Pharmaceutical industry under pressure}

Over 400 new medicines received approval between 2000 and 2012 [3]. In 2013, although more than 5000 medicines were in development globally, only 25 innovative new medicines [known as new molecular entities (NMEs)] and two biologics were approved by the FDA, accounting for $31 \%$ of the decline in approvals from 2012 [7]. Interestingly, many of the 2013 approvals offer exceptional earning potential. Indeed, $48 \%$ of the new approved medicines are set to achieve over US\$1 billion in annual sales within 5 years [7]. There is renewed hope because much of the pharmaceutical industry began to show signs of recovery in the second half of 2013 sales and earnings. Nevertheless, from a global point of view, the sales and earnings in 2013 of big pharmaceutical companies continued to decline, down by $1 \%$ and $3.2 \%$, respectively; whereas those of biotech companies rose by $12.6 \%$ and $12.9 \%$, respectively [8], and returns on R\&D investments continue to fall [9].

Importantly, 2014 was a good year for pharmaceutical productivity with $31 \mathrm{NME}$ and 11 biologic license applications, representing the best record since 1996. The FDA-approved drugs include eight cancer drugs (18\%) and 12 drugs that are broadly distributed against infectious diseases (27\%), four of which are antibiotics. Seventeen approved drugs showed novel mechanisms-of-action. About $65 \%$ of drugs were in-licensed or acquired and about $40 \%$ of the 2014 FDA-approved drugs target rare diseases (five approved drugs, $11 \%$ ). The aggregated sales were US\$53 billion and US\$48 billion in 2013 and 2014, respectively [10]. In short, their business relies on a costly, risky and timeconsuming hunt for novelty in drug candidates (first-in-class medicines to treat diseases with as-yet no treatment), genes and targets. New drugs must be more efficacious than those currently on offer, 
and should permit, for instance, shorter hospital stays to justify a higher cost to third-party payers. In spite of the forced reduction in R\&D productivity and the eroding revenues from established drugs owing to strong generic competition (small organic molecules) and in the near future to biosimilar competition [i.e. the antibody ( $\mathrm{Ab}$ ) field], the pharmaceutical industry needs to produce sustainable revenues and returns on investment for shareholders, which partly explains its addiction to blockbuster drugs. Concerning the small organic molecules, the generic share of prescriptions rose from $54 \%$ in 2003 to $84 \%$ in 2012, and will rise about $87 \%$ by 2017 [12]. In 2013, 16 biosimilar products were under development [13]. The first biosimilar monoclonal antibody (mAb) approved by European Medicines Agency (EMA) regulators was Inflectra ${ }^{\mathrm{TM}}$ which is a copy of Johnson \& Johnson's infliximab used to treat several inflammatory diseases (e.g. psoriasis, rheumatoid arthritis, etc.) [14]. After several years of debate, the FDA has approved Sandoz's Zarxio ${ }^{\mathrm{TM}}$. Zarxio ${ }^{\mathrm{TM}}$ is the first biosimilar drug available in the USA, is a copycat version of Novartis's Neupogen ${ }^{\circledR}$ and is a granulocyte colony-stimulating factor (G-CSF) analog used to stimulate the proliferation and differentiation of granulocytes [15].

\section{Scientific and strategic challenges for pharmaceutical industries: an overview}

Over the past decade, the drug industry has faced two major challenges, one scientific and one strategic. The scientific challenge concerns the translation from genomic information gathered to understand disease predisposition and progression as well as to predict a patient's response to drugs [16]. Thus, over the past several years, a strong emphasis has been given to the development of genomic and bioinformatics approaches to identify new biomarkers [17] or candidate drug targets [18]. This genomics and proteomics revolution has produced thousands of new targets for drug discovery [19]. Biomarkers can be considered as parameters or indicators that can be used to measure and evaluate: (i) normal biological processes; and (ii) the effects on biological systems of several agents such as drugs, as well as biological or physical agents. For instance, in oncology the main biomarkers are proteins [e.g. prostate-specific antigen (PSA), human epidermal growth factor (HER2), CK7], circulating tumor cells (e.g. p53), mRNA, radiological analysis [e.g. positron emission tomography (PET) scanning, molecular resonance imaging (MRI)], among others [20]. The use of biomarkers in Phase $1 / / 1$ trials to select patients can accelerate anticancer drug development [21].

Based on the analysis of 113 first-in-class drugs, between 1999 and 2013 the majority had been discovered using a target-based approach ( 71\%: 41\% small molecules; $30 \%$ biologics) versus systems-based approaches ( 30\%: 23\% chemocentric; $7 \%$ phenotypic screening). First-in-class drugs can be classified as follows: systems-based compounds comprising small molecules ( $50 \%)$ and natural products or substances ( 37\%); or target-based compounds comprising biologics $(\sim 42 \%)$, small molecules ( $38 \%)$ and natural products or substances ( $20 \%)$. The major drug-targeted families, G- protein-coupled receptors (GPCRs), kinases, proteases and ion channels are absent or underrepresented among drugs discovered via systems-based approaches [22]. Currently, the main different approaches to obtain chemical leads that are fully integrated in the early drug discovery processes are from natural products, from HTS, from existing drugs and from fragment-based discovery (FBDD) [23].

Generally speaking, drugs can fail in clinical trials or have delayed market entry as a result of the display of detrimental or fatal side-effects, which can be considered as a balance between different biological systems. The challenge is to analyze the side-effects globally and not only at the cellular, organ and human patient levels. For decades, the pharmaceutical industry has been actively working to drive down the important cost of drug failure in terms of time and money. Indeed, implementing strategies to identify the failures as early on in the process as possible is absolutely crucial, because failures during early stages are less costly than those at Phase III. Despite best efforts, failures in clinical trials have soared over the past 20 years, with attrition rates between 1990 and 2010 increasing for Phase I from 33\% to 46\%, for Phase II from $43 \%$ to $66 \%$ and for Phase III from $20 \%$ to $30 \%$. The current reasons for failure at Phase II are insufficient efficacy (51\%), safety concerns 
(19\%), strategic issues (29\%) and pharmacokinetics/bioavailability issues $(1 \%)$; and reasons for Phase III failures are insufficient efficacy $(66 \%)$, safety concerns $(21 \%)$, financial or commercial concerns $(7 \%)$ as well as non-disclosed issues (6\%). Generally speaking, new drug success rates in Phase II are about $20 \%$ (51\% insufficient efficacy and $19 \%$ safety concern) and about $50 \%$ in Phase III (66\% insufficient efficacy and $21 \%$ safety concerns) [24]. During R\&D, success at each step is mandatory, but 'good' earliest strategic choice remains crucial. Figure 1 highlights the main reasons for clinical failures by Phase for 410 drugs that entered human testing between 2000 and 2009 [25]. The main failures in Phase II and III studies are efficacy issues, $54 \%$ and $52 \%$, respectively. Safety issues represent about one-third of all the $\mathbf{4 1 0}$ drugs analyzed in Phase I and Phase III studies, versus $17 \%$ of all Phase II studies.

\section{New strategic vision in drug discovery}

\section{General aspects}

The development of a new and sustainable funding model with public and academic participations has become an urgent requirement [26]. To address this, new hubs are emerging that group together different research systems including academia, research institutes, the American National Institutes of Health (NIH) and the pharmaceutical industry, including biotechnology companies. Their aim is to put an end to these different sectors, each with their own drug-finding processes and objectives, working alone in their respective corners. Indeed, until recently, the motto 'every man for himself' has led to a lack of shared capitalization on collective scientific and technological knowledge and knowhow [27]. Much enthusiasm now surrounds an emerging translational research concept referring to the novel integration of discoveries into a common framework that could change the landscape of drug discovery [28]. Although still in its infancy, a recent article by Fishburn analyzed the roles taken by the different 'actors' and their respective contributions and benefits to the collective organization with the common goal of increasing the number of drugs successfully brought to market [29]. This study found that the most important investments are currently made by pharmaceutical companies and the NIH.

\section{Doing the right things or doing things right}

In this direction, Elebring [30] recently published a very instructive article that addressed the crucial question distinguishing the most important approach in current drug discovery: are we doing the right things or doing things right? The author found that the key points to consider in the drug discovery process are the right pharmacologic target and the right chemical lead. The major conclusion was that the quality of R\&D and drugs can reduce timelines and costs. We fully agree that the right selection of these two key elements ensure an improved R\&D productivity and the implementation of robust pipelines, whatever the strategy used: target-based screening or phenotypic screening approaches. Based on this principle, in Figure 2 we propose - like a 'mantra' for R\&D - a discipline for pharmaceutical R\&D aimed at improving productivity and efficiency in the generation of new drugs as a marriage between quality, speed and cost - developed by Elebring [30] - and druggability and quality of the clinical candidate, druggability and quality of the target and, the final objective, clinical efficacy of the drug. We fully agree with the comments of Elebring: 'Focus on science quality should lead to improved drug discovery quality, cost and speed' [30]. The high-quality of the bioactive molecule can be evaluated using several criteria such as e-ADME profile including PK/PD behaviors, metrics, drug-like concept [31], among others; whereas the target must be validated using several techniques [32]. The improvement of the quality of the target and bioactive molecule decrease the probability of failure in clinical trials. Reduction of time and cost $(R \& D)$ are strongly related to the high-quality of science. Recently, Kinch highlighted target selection for FDA-approved medicines [33]. 


\section{Open innovation as a model to find and to develop new drugs}

Twenty years ago, drug discovery was exclusively conducted within pharmaceutical organizations. Today, in the open innovation model, pharmaceutical companies commercialize not only their own ideas but also a considerable proportion of inventions and innovations from external organizations. One of the major challenges of the open innovation era is ensuring its strong integration within each of the different partners and a high level of coordination [34]. By encouraging a dynamic innovative environment with strong collaborations among pharmaceutical industries, academia and other industries, the open innovation mindset is a key to developing sources of solutions. Four new types of open innovation, 'knowledge creation', 'knowledge integration', 'knowledge translation' and 'knowledge leverage' have been described by Schuhmacher et al. [27]. The development of nanopharmaceuticals under open innovation is a good example of this new approach and has been analyzed by Eaton [35].

\section{Strategic mergers and acquisitions in the pharmaceutical and biotech industries}

The losses of the major revenue as a result of patent expirations have forced big pharmaceutical companies to scale patent cliffs and to look to biotech companies and others to: (i) fill in their pipeline; (ii) create consolidation purpose; (iii) achieve R\&D cost reduction; (iv) produce synergies; and (v) create a breakthrough innovation, but with important social consequences (i.e. job cuts, psychological disorders, etc.) [36]. Thus, for instance, Merck will cut 8500 jobs by the end of 2015 saving US\$2.5 billion [37]. The recent expiration of the blockbuster drug patents are as follows: Plavix ${ }^{\circledR}$ (clopidogrel), Lipitor ${ }^{\circledast}$ (atorvastatin calcium) and Seroquel ${ }^{\circledR}$ (quetiapine fumarate), inducing massive drops in revenue of US\$33 billion [38].

Today, half of all drugs are developed through collaborations, one-quarter of which are part of a merger, acquisition or co-development arrangement. In addition, $49 \%$ of pharma drug development arrangements are licensing, 25\% co-development, $24 \%$ merger and acquisition and $2 \%$ joint ventures [39]. An analysis performed by Elvidge showed the decline of the megadeals and the development of late-stage deals. More than $60 \%$ of the deals have been performed by big pharmaceutical companies [40]. For an interesting example, three leading pharmaceutical firms (Novartis, GSK and Eli Lilly) are set to exchange business segments in deals affecting at least 14000 employees, to reinforce their strongest businesses, Novartis in oncology, GSK in vaccines and Eli Lilly in animal health, and exit weaker ones [41]. In addition a joint venture has been set-up between GSK and Novartis focusing on cancer immunotherapy, epigenetics and tumor microenvironment [42]. Based on the interesting analysis of Kinch et al. [43], the evolution of major organizations - mainly business entities - controlling NMEs has been highlighted in Figure 3. From 1930 to 1960 the steady increase of the number of approved NMEs correlated with the proportional expansion of the organizations. From 1960 to 1980 the number of NMEs increased whereas the number of organizations that had obtained NMEs reached a plateau, with few new entries or exits. Starting in the 1980s, prominent change in drug discovery and development was observed. New companies such as start-up companies and biotechnology companies have seen the day and, at the same time, exits were largely caused by consolidation strategies (mergers and acquisitions) rather than bankruptcies.

\section{Strategic generics and biosimilars in the pharmaceutical industry}

Generic copies are pharmaceutically equivalent to the originator medicine, and represent a new vision in the pharmaceutical industry [12]. The market boom for generics as a result of the patent expiration (off-patent - the so-called patent cliff) has led to an abrupt drop in sales of medicines such as blockbusters. In the USA, they represented $19 \%$ and $86 \%$ in 1984 and 2013 , respectively, and represent the pillar of healthcare, with US\$1.5 billion of savings. Interestingly, in 1990, 50\% of the new drug applications were from the USA and $15 \%$ were from India, whereas today $30 \%$ are from the USA and $40 \%$ are from India. Figure 4 represents the main suppliers of generic drugs and the 2013 generic drug sales. According to Frost \& Sullivan's report, between 2010 and 2017 
pharmaceutical products worth US\$150 billion will go off-patent. The generic drugs market revenues will grow from $\sim$ US\$124 b illi o $n$ (2010) to US\$231 billion (2017) with strong generic drug manufacturer competitions. The manufacturers Teva, Sandoz and Mylan decided to produce higher value generics. Another strategy adopted by generic drug manufacturers is to set-up alliances with branded pharma for marketing and exclusivity rights to generic blockbusters such as Lipitor $^{\circledR}$ and Crestor $^{\circledR}$.

Biosimilars are revolutionizing the bioprocessing industry as functional replicas (antibody copy) of the originator biologic drugs [44]. The biosimilar market shows significant growth, mainly because of the launch in the next few years of numerous mAb biosimilars of mAbs where the patent will expire such as trastuzumab (Herceptin ${ }^{\circledR}$ from Roche) and adalimumab (Humira ${ }^{\circledR}$ from Abbott). Already, the European regulators have approved in 2013 the first biosimilar mAb, Inflectra ${ }^{T M}$ (Hospira), which is the biosimilar of Johnson \& Johnson's Remicade ${ }^{\circledR}$ (infliximab) to treat several inflammatory diseases such as psoriasis and rheumatoid arthritis. In 2012, the sales of Remicade ${ }^{\circledR}$ were more than US\$8 billion worldwide. Today, nine biosimilars of Remicade ${ }^{\circledR}$ are under development, 16 for Humira ${ }^{\circledR}$ (adalimumab; inflammatory diseases), 21 for Enbrel $^{\circledR}$ (etanercept; inflammatory diseases), 34 for Rituxan ${ }^{\circledast}$ (rituximab; cancers and arthritis), 30 for Herceptin ${ }^{\circledast}$ (trastuzumab; cancers) and 16 for Avastin $^{\circledR}$ (bevacizumab; cancers). The global biosimilars market revenue was estimated at around US\$172 million in 2010, and should be more than US\$4 billion in 2017, with an annual growth rate of about 56\%. The European Union (via the EMA), is the first region to adopt legislation allowing the registration of biosimilars based on biosimilar guidelines related to similarity to the reference product. Very recently, the FDA approved Sandoz's Zarxio ${ }^{\mathrm{TM}}$ and, interestingly, generics of nanodevices in medicine [45].

\section{Strategic visions to find and develop medicines}

\section{Has the modulation of a network of targets outdated the one-disease-one-target philosophy as the best strategy to find new drugs?}

A new paradigm is emerging in pharmacological research called polypharmacology, which describes the activity of compounds at multiple targets. The multifaceted goal is to avoid adverse side- effects (safety parameters), and to improve therapeutic efficacy, prevent drug resistance or reduce therapeutic-target-related adverse side-effects (efficacy parameters). As indicated by Peters, since the 1990s the pharmaceutical industry has focused its attention on searching for highly selective drugs - at the early stages of drug discovery - to avoid adverse side-effects [46]. Lack of selectivity was until then usually discovered later on in a drug discovery project. Generally, multitarget drugs - in combination or not - are more efficacious than single-target drugs, for instance in oncology and against viral infection. Furthermore, rather than a one-target therapy, polypharmacological modulation of a network of targets is actually required in the treatment of many multigenic diseases. For instance, multikinase inhibitors can block multiple targets in parallel signaling pathways and thereby prevent drug resistance caused by mutations or expression changes [47].

\section{First-in-class versus best-in-class and phenotypic- versus target-based drug discovery: what matters for commercial success?}

The development of genomics and proteomics has produced a plethora of targets for drug discovery. The main challenges are the validation of these new targets in specific human disease [48], and then the efficacy of the corresponding treatment in humans. The pharmaceutical industry is facing a challenge of productivity in R\&D. Thus, innovation strategy in discovery represents the most powerful strategy to find new drugs [49]; for instance, based on the discovery of new targets to deliver more and better drugs in the pipeline. Ideally, the pharmaceutical industry focused on firstin-class products (hypothesis-driven) based on new targets, but this strategy alone is not enough to 
solve the current productivity gap. The scientific knowledge is fundamental to the discovery of 'first-inclass' medicines that are based on many different biochemical mechanisms mediating the drug response at the target [22]. In addition, projects based on new targets have less probability to reach the market than those based on known targets, and the later entrants - where research is based on known targets - displace the first-in-class products in the market place, even if the first entrant has the exclusivity free of competition during a period of time [50].

Generally speaking, the semi-empirical phenotypic-based discovery does not require understanding of the mechanism- of-action but the understanding of the biology related to the disease with the risk to develop compounds without any understanding of the mechanism-of-action to evaluate dose-response relationship. A target-based discovery approach is based on a clear understanding of the mechanism- of-action allowing the selection of the biomarkers for patient stratification, development of target therapy and combinations.

Innovation requires high investment in $R \& D$ and is time consuming. The late entrant (rival) developing a best-in-class strategy achieved major commercial success, such as the blockbusters atorvastin calcium (Lipitor ${ }^{\circledR}$ ) from Pfizer and lovastatin (Mevacor ${ }^{\circledR}$ ) from Merck. Pharmaceutical companies producing such first-in-class drug candidates often develop a 'follow-on' drug based on the more validated (with the first drug) target to reduce the risk of obtaining no drug approval. Figure 5 represents the evolution of the FDA-approved first-in-class and follower drugs between 1999 and 2013 analyzed by Swinney and Anthony and Eder et al.

In an early study, Swinney and Anthony emphasized that, between 1999 and 2008, based on the number of NMEs, phenotypic screening was the most successful strategy to find first-in-class drugs whereas target-based screening was the most efficient at finding follow-on drugs [51]. The mechanism- of-action used by the majority of small-molecule first-in-class NMEs involved enzyme inhibition or receptor modulation. An important conclusion is that phenotypic screening enabled $\mathrm{t} \mathrm{h}$ e discovery of biologically active compounds (drug leads and clinical candidates) that are more likely to have relevant mechanisms-of-action.

Another analysis by Eder et al. [22] on all the 113 FDA-approved first-in-class drugs between 1999 and 2013, shows that the majority had been discovered using a target-based approach ( 71\%: $\sim 41 \%$ small molecules; $\sim 30 \%$ biologics) versus systems-based approaches ( 29\%: $\sim 22 \%$ chemocentric; $\sim 7 \%$ phenotypic screening). First-in-class drugs can be classified as follows: systemsbased compounds comprising small molecules $(\sim 50 \%)$ and natural products or substances ( $37 \%)$; or target-based compounds comprising biologics ( $\sim 2 \%)$, small molecules $(\sim 38 \%)$ and natural products or substances ( 20\%). Chemocentric approaches represent the identification of active compounds from, for instance, plants and derivatives, microbial extracts, serendipitious findings, among others. Numerous examples of drugs already identified using the chemocentric approach can be highlighted such as aspirin, nitisinone, nelarabine and ezogabine. In addition, the authors highlighted that phenotypic screening represents not only an important source of new drugs but also the development of new biology and pharmacological tools.

Today, about $70 \%$ of drugs within the pipeline are potential first-in-class medicines, the proportion of which varies according to therapeutic field: neurology (84\%), cancer (80\%), psychiatry (79\%) and immunology (72\%) [52]. The major drug-targeted families, GPCRs, kinases, proteases and ion channels are absent or under-represented among drugs discovered via systems-based approaches. Currently, the main different approaches to obtain chemical leads that are fully integrated in the early drug discovery processes are: from natural products, from HTS, from existing drugs and FBDD. Another interesting analysis has been performed by Schulze and Ringel showing that it is the same to be first-in-class with a best therapeutic advantage or to be best-in-class - also with a best therapeutic advantage - but second within no more than 2 years after the launch of the first drug [53]. Moffat et al. highlighted that, in the oncology domain, the target-based discovery approaches remain the main strategy used, but phenotypic screening also contributed to cancer drug discovery, for example cytotoxics are drugs against mitotic arrest [54].

Recently, Zheng et al. presented an interesting review about the renewal of phenotypic screening, 
as a new momentum in drug discovery. The new applications of phenotypic screening of known drugs are: (i) to identify new indications especially for diseases without effective therapies; and (ii) to repurpose new screens for new target identification purposes [55]. Table 1 summarizes the average costs and the length of time it takes for a drug to be FDA-approved related to drug discovery strategies.

Figure 6 shows the crossroad between phenotypic (empirical) and target (molecular) based discovery strategies for best-in-class and first-in-class medicine discovery. All options are theoretically possible. Importantly, as postulated by Swinney [51], the unmet medical need should be tackled via the following chronological pathway: (i) phenotypic assay related to the unmet specific human disease affording first-in-class active derivatives; (ii) target identification process to link the specific mechanism-of-action and the phenotypic screening; and (iii) target-based discovery approach to find best-in-class drugs. This progression is correlated with the iterative increase in knowledge from empirical analysis (early stage) to strong knowledge of the specific molecular mechanism-of-action. In Figure 7 we present an overview of the main strategies developed to find and to produce new small-molecule drugs. These strategies are based on the knowledge or not of the target such as protein structure and the structure of the ligand related to this target. It is not in the scope of this review to present and to analyze the different strategies developed to find new medicines [56], but only to represent an overview.

\section{The future of pharmaceutical science related to industry and academia}

Interestingly, Crommelin et al. proposed four potential scenarios for the pharmaceutical sciences in 2020 based on the identification of four potential scenarios [57]. As shown in Figure 8, the matrix can be divided in four parts: (i) scenario 1 (pharmaceutical companies: innovation spirit; academia: public-private partnerships); (ii) scenario 2 (pharmaceutical companies: new types of businesses based on lifestyle technologies; academia: spins-off companies, public-private partnerships); (iii) scenario 3 (pharmaceutical companies: niche markets; academia: source of fundamental knowledge, few public-private partnerships); and (iv) scenario 4 (pharmaceutical industries: only clinical trial development corresponding to the decline of majors; academia: drug-finding strategy, few publicprivate partnerships). These four scenarios are at the crossroads of two aspects of the science: scientific culture (knowledge to develop and market new drugs) and treatment and prevention of the diseases (societal attitudes). In Figure 8 only the pharmaceutical and academic views are presented.

\section{An at-a-glance vision for new hit, lead and drug optimization processes using the space concept strategy}

The nearly infinite number of potential drugs present in the chemical space continuum of biological systems encompasses all possible organic molecules and is limited only by the chemist's imagination. The main challenge left to face in the discovery and development of new medicines is scouting the richly diverse chemical space in the search regions that contain specific biologically active derivatives with adequate physicochemical or topological properties. Since the introduction by Lipinski and Hopkins of the concept of navigation and exploration inside the less-well-trodden chemical spaces in biology and medicine, many efforts have been made to find and to develop original drugs [58]. The objective of medicinal chemistry is thus to expand the druggable chemical space by the identification of new regions, thereby paving the way to finding new drugs, in particular those required for challenging targets [59]. The concept of drug-like chemical space has widely been used in drug development for the selection of molecules to be screened, tested or selected, as well as for the assessment of the quality of the hit and lead compounds or final drugs. A far-ranging goal of this research is to identify new discrete chemical regions (called clusters, subtractions, etc.) to find and to develop original drugs, especially for challenging targets.

Inspired by the Lipinski and Hopkins concept of navigation and exploration of the chemical space, 
we propose in Figure 9 a simple overview for new hit, lead and drug optimization process using the space concept strategy and several metrics to qualify these different chemical entities. For instance, the virtual and real chemical space can be subdivided into four subareas (also known as clusters) associated with several specific characteristics. Thus, these four subset chemical regions have been parameterized using a large number of specific chemical and physicochemical properties or topological descriptors as recognition patterns. For example, a region might be described based upon its eADMET properties, drug-likeness in silico or Lipinski's rule-of-five (Ro5), indicating the possible oral bioavailability of drugs via passive absorption from the intestine. Importantly, Ro5 also determines the boundaries of spaces for appropriate potential druggable final drugs.

As shown in Figure 9, four clusters have been parameterized, for instance by $\mathrm{MW}$, clogP, number of hydrogen acceptors (NHA) and number of hydrogen donors (NHD), to define discrete areas such as the drug-like chemical space (Lipinski's rule-of-five, Ro5, oral route, grey cube), lead-like chemical space (Oprea's criteria, rule-of-four, Ro4, green cube), fragment chemical space (rule-of-three, Ro3, bright blue cube) and one mauve cube dedicated to the Ro5? for transdermal drugs. Other spaces dedicated to other administration routes can be used (mauve cube) [60].

Inside the Ro5 (clinical candidates), Ro5' (clinical candidates) and Ro4 (leads), first we recommend $L E$ as a simple indices or metric to quantify the molecular quality of the different chemical entity types, for selection and optimization inside each cluster. Other indices can be used such as LLE, BEI/SEI, SIHE or QED. QED is used only for clinical candidates. The optimization navigation process between each cluster can be performed using LE (Figure 9), LLE or BEI/SEI [61]. Based on these metrics, construction of more druggable libraries (from fragments, leads or drugs) can be developed.

The overlapping of the drug-likeness chemical space continuum (Ro5, grey cube) and the 3D target classes (block-cylinder, also called target space) including individually, for instance, PPI space, kinase space, GPCR space, defined an overlap volume (green rays, truncated cylinder) for which all the compounds (virtual or real) within this space are druggable; the anti-overlap area corresponds to the poorly druggable compounds. The same parameters used to define the boundaries of druggable compounds (e.g. Ro5) can be used to define a specific target space including drugs.

\section{Concluding remarks and future perspectives}

With our combined expertise gained in the pharmaceutical industry and academia, in this concise review, we present our common analysis on why and how drug discovery strategies in pharmaceutical companies changed. Drug discovery represents the first step in the creation of new drugs, and occurs in academic institutions, biotech companies and large-medium pharmaceutical companies. Throughout the past one-to-two decades, the decline in R\&D productivity, in other words the decrease in output of truly new drugs, has led to a pharmaceutical crisis. Multifaceted strategies have been implicated to overcome the pressure such as: (i) increased expenditure on R\&D; (ii) enhanced mergers, deals and acquisitions; (iii) reinforced product in-licensing capabilities; (iv) implementation and reorganization of new R\&D facilities to cater for new and powerful technologies such as computational chemistry, focused HTS, fragment approaches and genomics, proteomics or metabolomics approaches, among others; (v) development and implementation of tools that help remove part of the uncertainty element of pharmaceutical research, such as druggability prediction methods favoring production of high-quality active compounds, and new metrics of ligand efficiency; (vi) development of new pharmaceuticals to open up new markets, for example orphan drugs, personalized medicine; (vii) development of an open innovation strategy; and (viii) considerable staff cutbacks.

In this review we have analyzed the main new strategies in drug discovery aimed to disrupt the spiral of decline in R\&D productivity. We believe that the first step R\&D should be taking is to develop strategies to deliver high-quality clinical candidates based on druggable building blocks, fragments, hits and leads, for instance using fragment-based approaches. The second step to take 
would then be to develop druggable targets either from patients, using a biomarker strategy, or from validated targets to decrease the clinical attrition rate. Another direction is the development of personalized medicine associated with companion diagnostic tests not only in the domain of oncology but also in other therapeutic fields. We fully agree with the important point made by Elebring [30] that 'too much process thinking in drug discovery, such as Ro5, Ro3 etc., can block enthusiasm, creativity, intuition, innovation and serendipity'. Future success in drug discovery must therefore depend on achieving the correct balance between 'doing the right things and doing the things right'. Recently, Bennani [62] introduced a new concept, termed 'innovation ASAP', the expansion of which we believe would further enhance innovation culture - already a hot topic in many other industries - inside the pharmaceutical industry. The embracing of such a concept is required to stimulate the delivery of more and improved next-generation drugs through the pipeline for currently untreated diseases, and to shape the pharmaceutical R\&D business toward effective prosperity. The rethinking by the pharmaceutical industry of major innovation strategies, such as the implementation of the 'open innovation/disruptive innovation' principle across the entire R\&D process, represents one of the main goals toward achieving success.

Getting the right balance between first-in-class products based on new targets and second-in-class products, as well between target-based and phenotypic approaches, constitutes a lifeline offering the chance to resolve the drug pipeline attrition. In addition, to reduce risk of failure in early clinical trials and reduce production costs, pharmaceutical companies should invest more time in the development of the phenotypic screening approach as a repurposing screen of already approved drugs to identify: (i) new indications for diseases without an effective therapy; and (ii) new targets (rare or neglected diseases). The take home message should be that progress in drug discovery can be achieved with the association of three mindsets: information, education and collaboration.

\section{Acknowledgments}

J.R. and H.T. acknowledge the support of FCT - Fundação para a Ciência e a Tecnologia, under CQM grant PEst-OE/QUI/UI0674/2014 (CQM is a FCT National Research Unit).

\section{References}

1 Tollman, P. et al. (2011) Identifying R\&D outliers. Nat. Rev. Drug Discov. 10, 653-654

2 Scannell, J.W. et al. (2012) Diagnosing the decline in pharmaceutical R\&D efficiency. Nat. Rev.

Drug Discov. 1, 191-200

3. PhRAMA (2015) Biopharmaceutical research industry profile. Available at:

http://www.phrma.org/sites/default/files/pdf/2014_PhRMA_PROFILE.pdf

3 Arlington, S. and Davies, N. (2012) Diverging paths, difficult choices. Drug Discovery World.

Available at: http://www.ddw-online.com/business/p205977-diverging-pathsdifficult-choices-winter2012.html

4 IFPMA (2011) The pharmaceutical industry and global health: facts and figures. Available at: http://www.ifpma.org/fileadmin/content/Publication/2011/2011_The_Pharmaceutical_Industry_an d_Global_Health_low_ver2.pdf

5 Paul, S.M. et al. (2010) How to improve R\&D productivity: the pharmaceutical industry's grand challenge. Nat. Rev. Drug Discov. 19, 203-214 
6 Center for drug evaluation and research, FDA and US department of health and human services (2012) Impact, innovation, predictability, access. Available at:

http://www.fda.gov/downloads/Drugs/DevelopmentApprovalProcess/DrugInnovation/UCM337830. pdf

7 Mullard, A. (2014) 2013 FDA drug approvals. Nat. Rev. Drug Discov. 13, 85-89

8. Jarvis, L.M. (2014) Pharma growth still stagnant. Chemical Engineering News March 92, 22-24

9 News in Brief (2014) Returns on R\&D investments continue to fall. Nat. Rev. Drug Discov. 13, 9

10 1) Munos B., The FDA approvals of 2013: a watershed?, http://www.forbes.com/sites/bernardmunos/2014/01/03/the-fda-approvals-of-2013-a-watershed [accessed June 2015] 2) Mullard A. (2014) 2014 FDA drug approvals. Nature Rev. Drug Discov. 14, 7781 3) Jarvis L. M. (2015) The year in new drugs CEN, February 2015, 11-16

12 1) Thayler A. (2014) 30 years of generics, CEN, September 29, 8-16 2) Mullin R. (2012) Beyond the patent cliff, CEN, December 10, 1520 3) Latham, C. (2013) Generic outlook: turning to innovation after the patent cliff. Pharmacy Times. Published online http://www.pharmacytimes.com/print.php?url=/publications/issue/2013/Generic-Supplement2013/Generics-Outlook-Turning-to-Innovation-After-the-Patent-Cliff [accessed June 2015] 13 1) Thayer A. M. (2013) The new copycats, CEN October 2013, 15-23 2) Galbraith D. (2014) Biosimilars awaken CROs, BioProcess Int., 24-28 3) Moores, Y. (2014) Demonstrating biosimilarity: PK considerations, Appl. Clinic. Trials, October/November 2014, 28-35

14 News \& Analysis (2013) EMA backs approval of first monoclonal antibodies, Nature Rev. Drug Discov. 12, 568

15 1) Trager R. (2015) US poised to approve its first biosimilar, Chem. Word, February 2015, 15 2) Ledford H. (2015) First biosimilar drug set to enter US market. Nature, 17, 253-254

16 K. A. Phillips et al. (2008) Challenges to the translation of genomic information into clinical practice and health policy: utilization, preferences, and economic value. Curr. Opin. Mol. Ther., 10, 260-266

17 1) Frank R. \& Hargreaves R. (2003) Clinical biomarkers in drug discovery and development. Nature Rev. Drug Discov. 2, 566-580 2) Tan D. S. W. et al. (2009) Biomarker-driven early clinical trials in oncology: a paradigm shift in drug development. Cancer J., 15, 406-420 3) Carden C. P. et al. (2010) Can molecular biomarker-based patient selection in Phase I trials accelerate anticancer drug development?. Drug Disc. Today, 15, 88-97 4) Bonnefoy J-Y. (2008) The biomarker revolution: a step toward personalized medicine. Pers. Med., 5, 553-556

18 Moos W. H. (2007) Comprehensive medicinal chemistry II, The intersection of strategy and drug research. Ed. Taylor J. B. \& Triggle, D. J. Elsevier, 1-64 
19 Plebani M. (2005) The next revolution in laboratory medicine?. Clin. Chim. Acta, 357, 113-122

20 Kloth M. \& Buettner R. (2014) Changing histopathological diagnostics by genone-based tumor classification. Genes, 5, 444-459

21 Carden C. P. et al. (2010) Can molecular biomarker-based patient selection in Phase I trials accelerate anticancer drug development. Drug Disc. Today, 15, 88-97

22 1) Eder J. et al. (2014) The discovery of first-in-class drugs: origins and evolution. Nature Rev. Drug Discov. 13, 577-587 2) Schulze U. \& Ringel M. (2013) What matters most in commercial success: firstin-class or best-in-class?. Nature Rev. Drug Discov. 12, 419-420 3) Booth B. \& Zemmel R. (2003) Quest for the best. Nature Rev. Drug Discov. 2, 838-841

23 Pors K. (2011) Drug Discovery into the 21st Century, Drug Discovery and Development - Present and Future, Ed. Dr. Izet Kapetanović, InTech Publishers

24 1) Arrowsmith J. (2011) Trial watch: Phase II failures: 2008-2010, Nature Rev. Drug Discov. 10, 328-329 2) Arrowsmith J. (2011) Trial watch: Phase III failures: and submission failures: 2007-2010, Nature Rev. Drug Discov. 10, 87 
25 Tufts Center for the Study of Drug Development, Data analysis. Reasons for clinical failures by phase (December 2013/January 2014), Appl. Clin. Trials (page 12)

26 Moors E. H. M. et al. (2014) Towards a sustainable system of drug development, Drug Discov. Today, 19, 1711-1720

27 Schuhmacher A. et al. (2013) Models for open innovation in the pharmaceutical industry, Drug Discov. Today, 18, 1133-1137

28 Feuerstein G. (2010) A translational medicine model in drug discovery and development, Int. Drug Discovery, April/May 2010, 30-33

29 Fishburn S. (2013) Translational research: the changing landscape of drug discovery, Drug Discov. Today, 18, 487-494

30 Elebring T. (2012) What is the most important approach in current drug discovery: doing the right things or doing the things rights? Drug Discov. Today 17, 1166-1169

31 1) Leeson P. (2012) Drug discovery: Chemical beauty contest. Nature 481, 455-456 2) Mirza A. et al. (2009) Known drug space as a metric in exploring the boundaries of drug-like chemical space. Eur. J. Med. Chem. 44, 5006-5011

32 Sams-Dodd F. (2005) Target-based drug discovery: is something wrong? Drug Discov. Today, 18, 139-147

33 Kinch M. S. (2015) Target selection for FDA-approved medicines, Drug Discov. Today, Available online 17 February 2015

34 1) Simpson P. B. \& Reichman M. (2014) Opening the lead generation toolbox, Nature Rev. Drug Discov. 13, 3-4 2) Judd D. B. (2013) Open innovation and intellectual property: time for a reboot? Drug Discov. Today, 18, 907-909 3) Aronson J. K. et al. (2012) Defining rewardable innovation in drug therapy, Nature Rev. Drug Discov. 11, 253-254 4) Niedergassel B. \& Leker J. (2009) Open innovation: chances and challenges for pharmaceutical industry, Future Med. Chem., 1, 119-1200

35 Eaton M. A. W. (2011) How do we develop nanopharmaceutical under open innovation? Nanomed-Nanotechnol, 7, 371-375

36 LaMattina J. L. (2011) The impact of mergers on pharmaceutical R\&D, Nature Rev. Drug Discov. $10,559-560$

37 Extance A. (2013) Merck to cut 8500 more jobs by 2015, Chem. World, November 2013, 16

38 Harrison C. (2013) Dangling from the patent cliff, Nature Rev. Drug Discov. 12, 14-15

39 1) Types of pharma drug development arrangements (2014) Appl. Clin. Trials (October/November 2014) 8 2) Half of all drugs today are developed through collaborations (2014) Appl. Clin. Trials, June/July 2014, 16 
40 Elvidge S. (2012) The changing shape of dealmaking, Biopharmadealmarkers, available at http://www.atebion-bds.com/pdfs/changing-shape-of-dealmaking.pdf [accessed February 2015]

41 1) Extance A. (2014) \$25bn trade sharpens pharma focus, Chem. World, June 2014, 16 2) Thayer A. (2014) Pharma firms swap assets, CEN, April 28, 5

\section{PMLive}

http://www.pmlive.com/pharma news/novartis pays $\$ 16 \mathrm{bn}$ for gsks oncology portfolio 562086 [accessed June 2015]

43 Kinch M. S. et al. (2014) An overview of FDA-approved new molecular entities: 1827-2013, Drug Discov. Today, 19, 1033-1039

44 1) Thayer A. (2013) The new copycats, CEN, October 7, 15-23 2) Moores Y. (2014) Demonstrating biosimilarity: PK consideration, Appl. Clin. Trials, October/November 2014, 28-35 3) Market boom for generics (2012), Pharm. Technol. Eur. (March 2012), 8

45 Davenport M. (2014) Covering the spread, CEN, November 10, 10-13

46 1) Peters, J-U. (2013), Polypharmacology - Foe or Friend?, J. Med. Chem. 56, 8955-8971 2) Bickerton G. R. et al. (2012) Quantifying the chemical beauty of drugs. Nature Chem. 4, 90-98 3) Proschak E. (2013) Reconsidering the drug discovery pipeline for designed multitarget drugs, Drug Discov. Today, 18, 1129-1130

47 Azmi, A. S. (2012) Network pharmacology for cancer drug discovery: are we there yet? Future Med. Chem., 4, 939-941

48 Plenge R. M. et al. (2013) Validating therapeutic targets through human genetics, Nature Rev. Drug Discov. 12, 581-594

49 Aronson J. K. et al. (2012) Defining rewardable innovation in drug therapy, Nature Rev. Drug Discov. 11, 253-254

50 Baker A. \& Gill J. (2005) Rethinking innovation in pharmaceutical R\&D J. Commerc. Biotechnol., 12, 45-49

51 1) Swinney D. C. \& Anthony J. (2011) How were new medicines discovered?, Nature Rev. Drug Discov. 10, 507-519 2) Swinney D. C. (2013) Phenotypic vs. target-based drug discovery for first-inclass medicines, Clin. Pharm. and Ther., 93, 299-301

52 PhRMA. Available at http://fr.slideshare.net/PhRMA/phrma-chart-pack-april-2013 [accessed June 2015]

53 Schulze U. \& Ringel M. (2013) What matters most in commercial success: first-in-class or best-inclass?, Nature Rev. Drug Discov. 12, 419-420

54 Moffat J. G. et al. (2014) Phenotypic screening in cancer drug discovery - past, present and future, Nature Rev. Drug Discov. 13, 588-602

55 Zheng W. et al. (2013) Phenotypic screens as a renewed approach for drug discovery, Drug Discov. Today, 18, 1067-1073 
56 1) Aboud-Garbia, M., (2009) Discovery of innovative small molecule therapeutics, J. Med. Chem., 52, 2-9 2) Hoelder, S., Clarke, P. A., Workman, P. (2012) Discovery of small molecule cancer drugs: successes, challenges and opportunities, Mol. Oncol., 6, 155-176

57 Crommelin D. et al. (2010) Pharmaceutical sciences in 2020, Nature Rev. Drug Discov. 9, 99-100

58 Lipinski C. \& Hopkins A. (2004) Navigating chemical space for biology and medicine, Nature, 432, 855-861

59 1) Keller T. H. et al. (2006) A practical view of 'druggability', Curr. Opin. Chem. Biol., 10, 357-361 2) Yusof I. \& Segall M. D. (2013) Considering the impact drug-like properties have on the chance of success, Drug Discov. Today, 18, 659-666

60 1) Teague S. J. et al. (1999) The Design of Leadlike Combinatorial Libraries. Angew. Chem. Int. Ed. 38, 3743-3748) 2) Oprea T. I. et al. (2001) Is there a difference between leads and drugs? A historical perspective. J. Chem. Inf. Comput. Sci. 41, 1308-1315 3) Oprea T. I. (2002) Current trends in lead discovery: are we looking for the appropriate properties. J. Comput. Aided Mol. Des., 16, 325-334 4) Makara G. M. (2007) On Sampling of Fragment Space. J. Med. Chem., 14, 3214-3221 5) Paern J. et al. (2007) Exploring fragment spaces under multiple physicochemical constraints. J. Comput. Aided Mol. Des., 21, 322-340 6) Goldberg F. W. et al. (205) Designing novel building blocks is an overlooked strategy to improve compound quality. Drug Discov. Today, 20, 11-17

61 1) Hopkins A. L. et al. (2014) The role of ligand efficiency metrics in drug discovery, Nature Rev. Drug Discov. 13, 105121 2) Abad-Zapatero C. et al. (2010) Ligand efficiency indices for an effective mapping of chemico-biological space: the concept of an atlas-like representation, Nature Rev. Drug Discov. 15, 804-811

62 Bennani, Y.L. (2011) Drug discovery in the next decade: innovation needed ASAP. Drug Discov. Today 16, 779-792

\section{Figure legends}

Figure 1. Main reasons for clinical failures by Phase based on 410 drugs that entered human testing between 2000 and 2009. The main failures in Phase II and III studies are efficacy issues, $54 \%$ and $52 \%$, respectively. Safety issues represent about one-third of all the 410 drugs analyzed in Phase I and Phase III studies, versus $17 \%$ of all Phase II studies.

Figure 2. Inter-related core aspects for the 'mantra' of R\&D for key decisions in drug discovery. Efforts to improve quality of science for drugs and targets, for instance based on translational biology, biomarkers from patients corresponding to a specific target and druggability for candidate drugs, should reduce timelines and cost, and increase the successes in clinical trials.

Figure 3. Evolution and changes of key organizations versus NMEs (new molecular entities). From 1930 to 1960 the increase of the number of approved NMEs is strongly correlated with the proportional expansion of the organizations; whereas from 1960 to 1980 the number of NMEs increased, and the number of organizations that had obtained NMEs reached a plateau, with few new entries or exits. Starting in the 1980s, prominent change in drug discovery and development was observed. New companies such as start-up companies and biotechnology companies expired and, at the same time, exits were largely caused by consolidation strategies (mergers and 
acquisitions) rather than bankruptcies.

Figure 4. Top generic firms, data from [12].

Figure 5. Percentage evolution of new molecular entities (NMEs) in each category of drug class (1999-2013), based on approved first-in-class and follower drugs. Between 1999 and 2008 phenotypic screening was the most successful strategy to find first-in-class drugs, whereas target-based screening was the most efficient at finding follower drugs. From 1999 to 2013 the majority of NMEs had been discovered using a target-based (small molecules and biologics) versus systems-based approach (chemocentric and phenotypic screening).

Figure 6. Crossroad between phenotypic- and target-based discovery strategies and best-in-class and first-in-class to develop new drugs for unmet medical need. All options are theoretically possible. Swinney [51] (green arrow) postulated that the unmet medical need should be tackled via a process based on an iterative increase in knowledge, and the progression to find best-in-class drugs from empirical (phenotypic) to hypothesis (target-based approach) testing.

Figure 7. Main current strategies to find and develop new small molecule drugs. The red arrows represent the classical optimization process or the hit- or lead-finding process.

Figure 8. Potential scenarios of the pharmaceutical science based on Crommelin et al. analysis [57]. The matrix shows a global view of the potential scenarios.

Figure 9. Simple overview for new hit, lead and drug optimization process using the space concept strategy (real and virtual) and metrics to qualify chemical entities. The chemical space can be subdivided into four clusters associated with several specific chemical and physicochemical properties or topological descriptors as recognition patterns such as $\mathrm{MW}$, clogP, number of hydrogen acceptors (NHA) and number of hydrogen donors (NHD) to define these discrete areas. Thus, drug-like chemical space (Ro5, oral route, grey cube), lead-like chemical space (Ro4, green cube), fragment chemical space (Ro3, bright blue cube) and one mauve cube dedicated to the Ro5' for transdermal drugs can be represented. Other spaces dedicated to other administration routes can also be used (mauve cube). Inside the Ro5 (clinical candidates), Ro5' (clinical candidates) and Ro4 (leads), the use of the ligand efficiency (LE) as a simple indices or metric to quantify the molecular quality of the different chemical entity types, for selection and optimization inside each cluster. Other indices can be also used such as LLE, BEI/SEI, SIHE or QED. As shown, the optimization navigation process between each cluster can be performed using LE, LLE or BEI/SEI. Based on these metrics, construction of more 'druggable' libraries (from fragments, leads or drugs) can be developed. The overlapping of the drug-likeness chemical space continuum (Ro5, grey cube) and the 3D 'target classes' (block-cylinder, also called target space) including individually, for instance, PPI space, kinase space, G-protein-coupled receptor (GPCR) space etc., defined an overlap volume (green rays, truncated cylinder) for which all the compounds (virtual or real) within this space are druggable; the anti-overlap area corresponds to the poorly druggable compounds. The same parameters used to define the boundaries of druggable compounds (e.g. Ro5) can be used to define a specific target space including drugs.

Table 1. Average of costs and FDA approval times according to drug discovery strategies

\begin{tabular}{|l|l|l|l|l|}
\hline & $\begin{array}{l}\text { Target-based drug } \\
\text { discovery }\end{array}$ & $\begin{array}{l}\text { Phenotypic-based } \\
\text { drug discovery }\end{array}$ & $\begin{array}{l}\text { Drug repurposing } \\
\text { screen }\end{array}$ & $\begin{array}{l}\text { Target } \\
\text { identification by } \\
\text { repurposing } \\
\text { screen }\end{array}$ \\
\hline FDA approval & $\sim 10-12$ years & $\sim 10-12$ years & $\sim 2-3$ years & $\sim 4-6$ months \\
\hline
\end{tabular}


$\square$ Safety $\square$ Efficacy $\square$ Commercial viability $\square$ Other issues

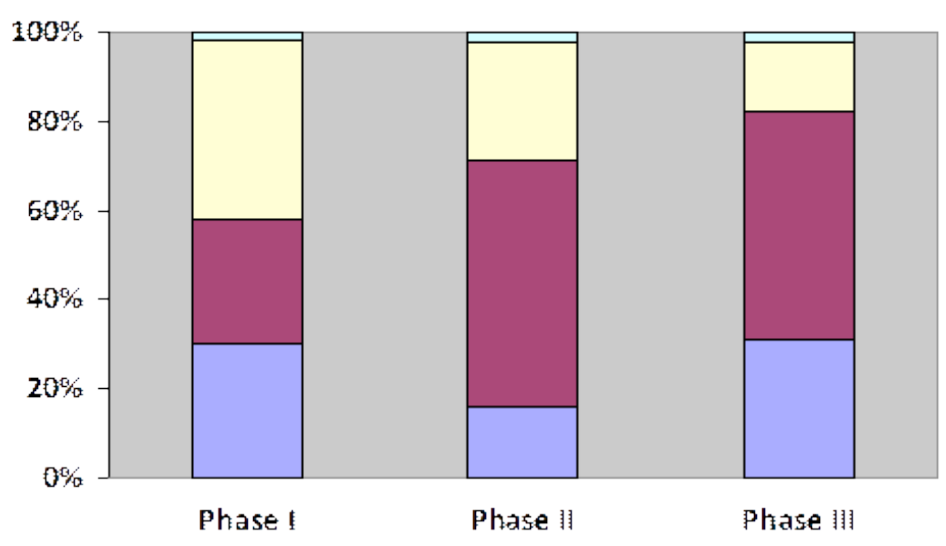

Figure 1. Clinical failures by clinical trial phases.

File: Image (GIF) 


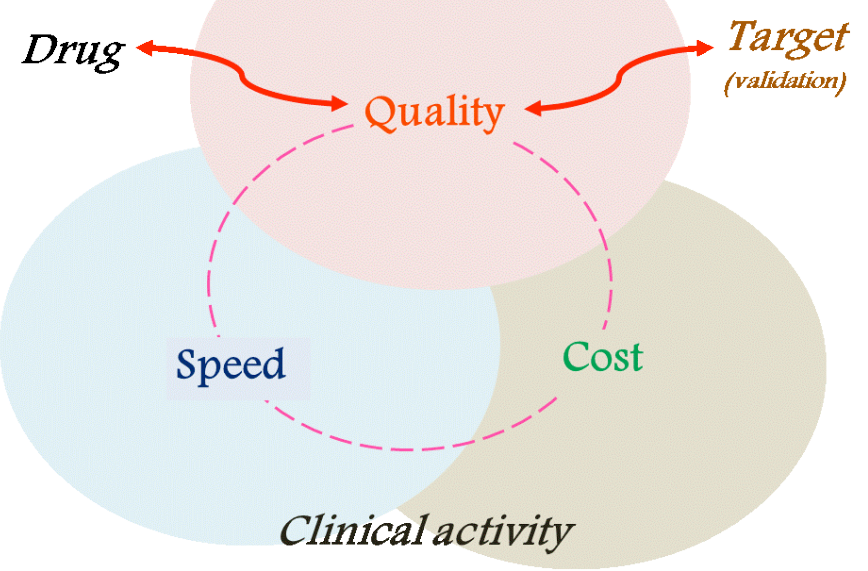

Figure 2. Inter-related core aspects for 'Mantra' of R\&D for key decisions in drug discovery.

File: Image (GIF) 


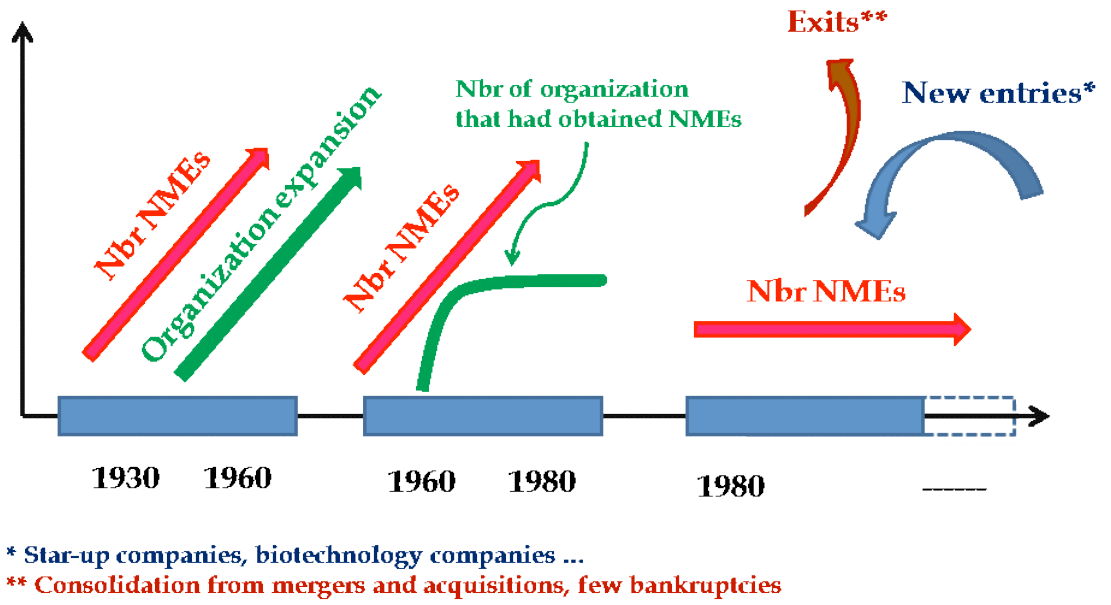

Figure 3. Evolution/changes of key organizations (NMEs: new molecular entities).

File: Image (GIF) 


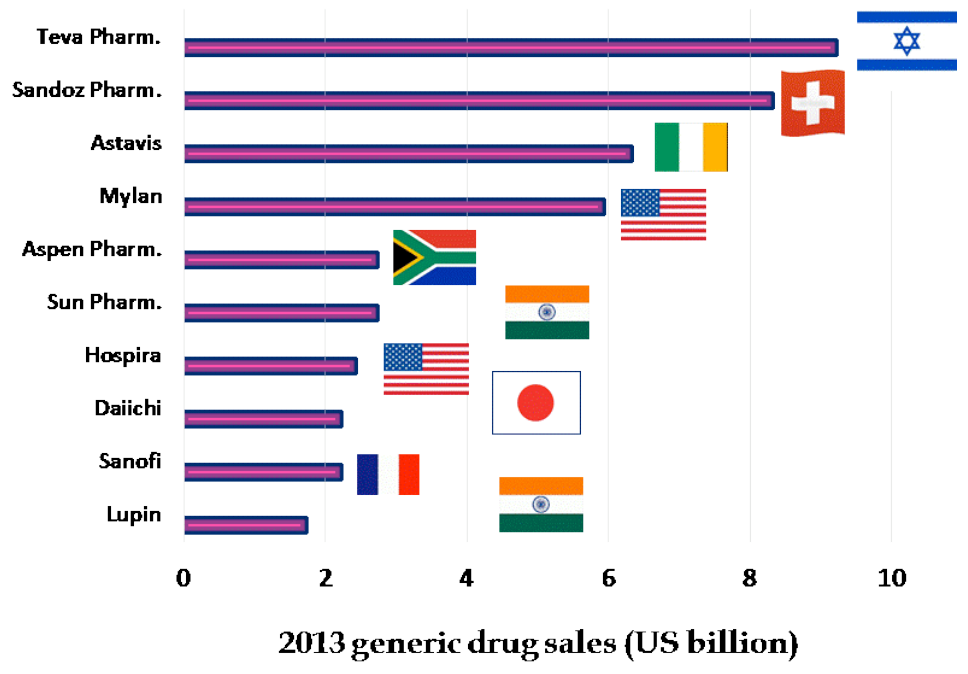

Figure 4. Top generic firms, data from ref. 13.

File: Image (GIF) 


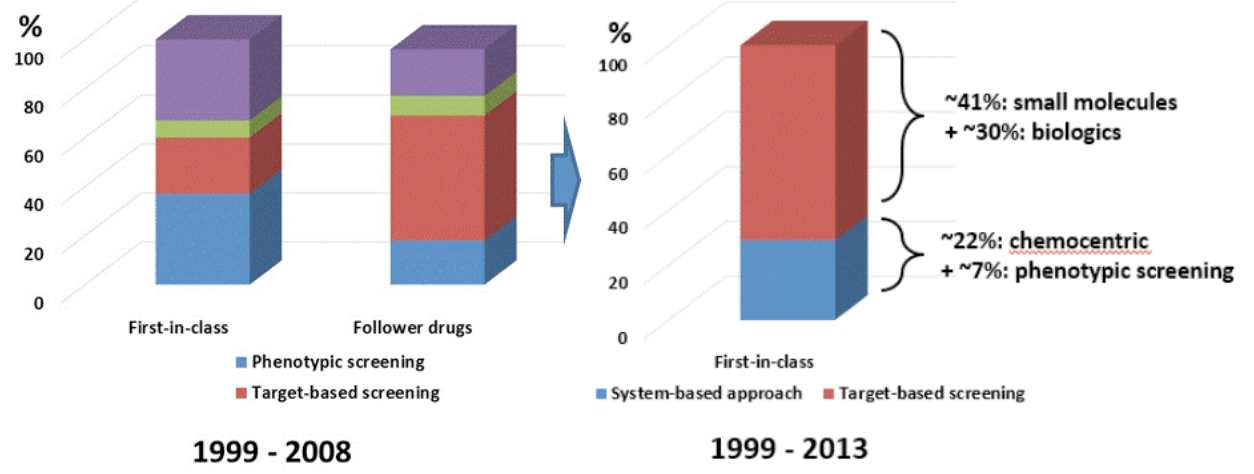

Figure 5. Percentage of NMEs in each category of drug class (1999-2013).

File: Image (GIF) 


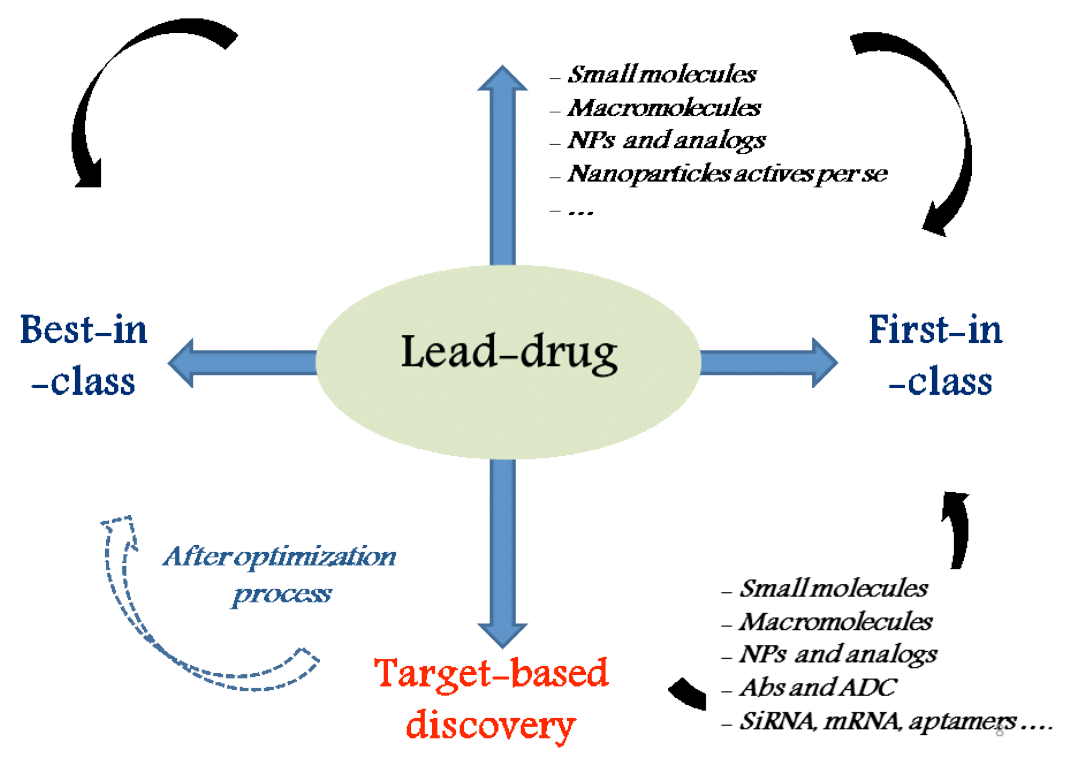

Figure 6. Cross road between phenotypic- and target-based discovery and best-in-class and first-in-class.

File: Image (GIF) 


\section{Protein* structure knowledge \\ Known \\ Unknown}

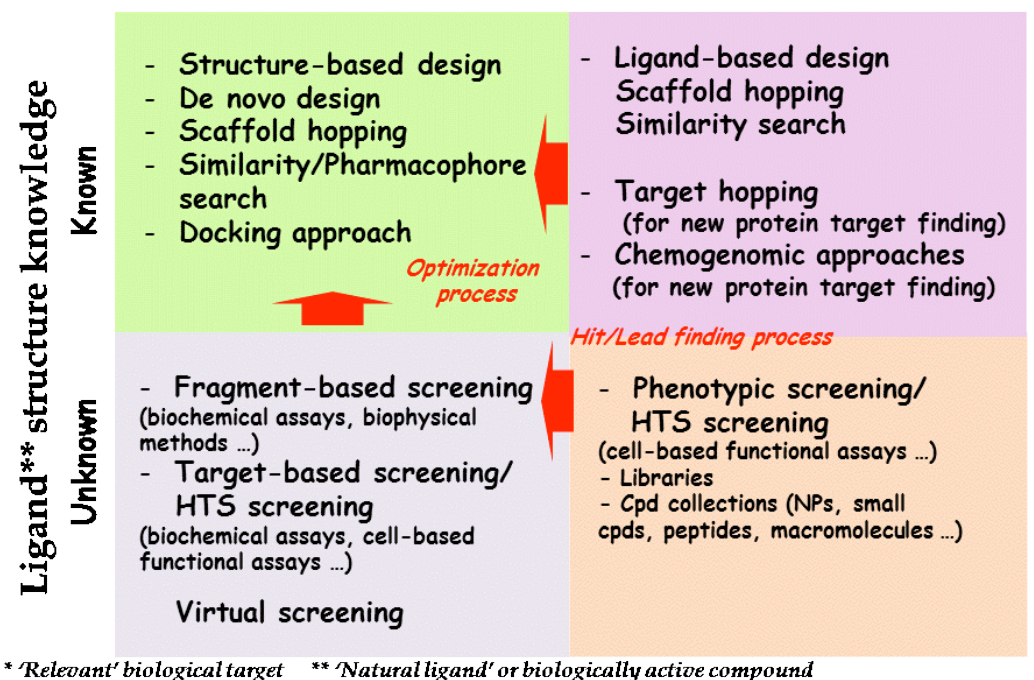

Figure 7. Main current strategies to find and develop new drugs. The red arrows represent the optimization process or the hit/lead finding process.

File: Image (GIF) 


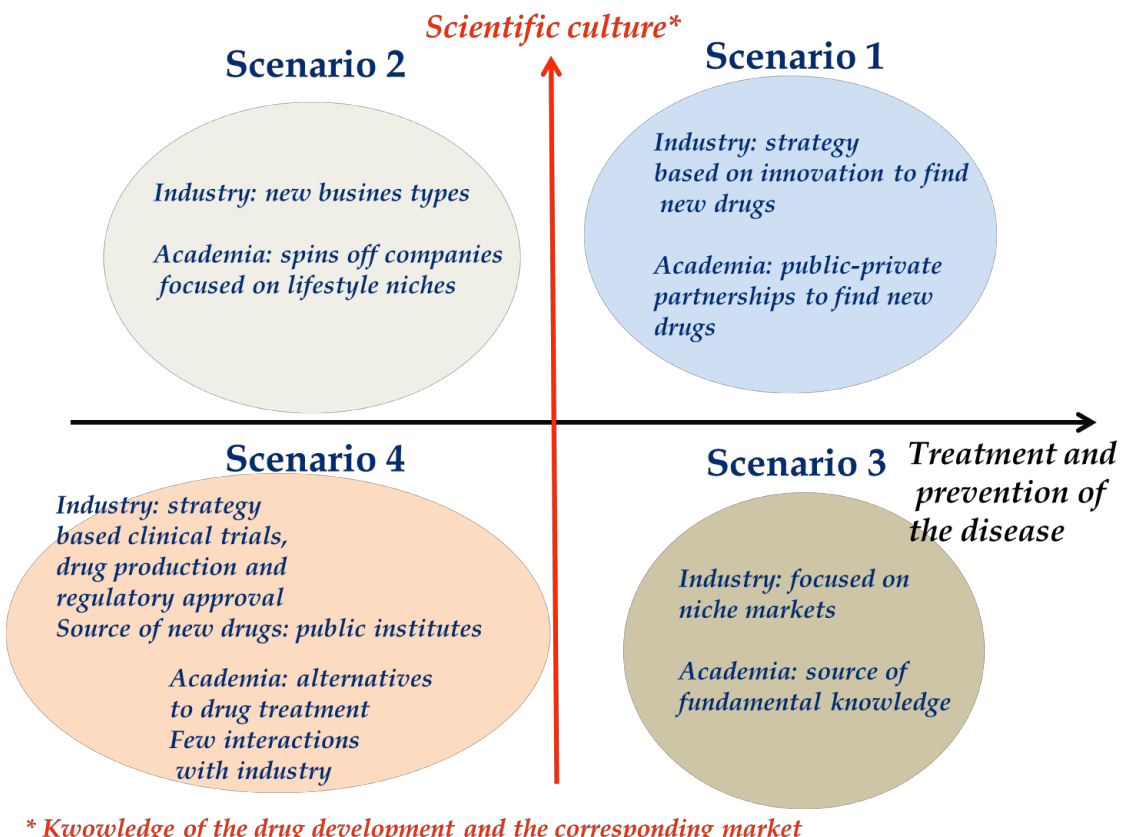

Figure 8. Potential scenarios of the pharmaceutical science.

File: Image (GIF) 


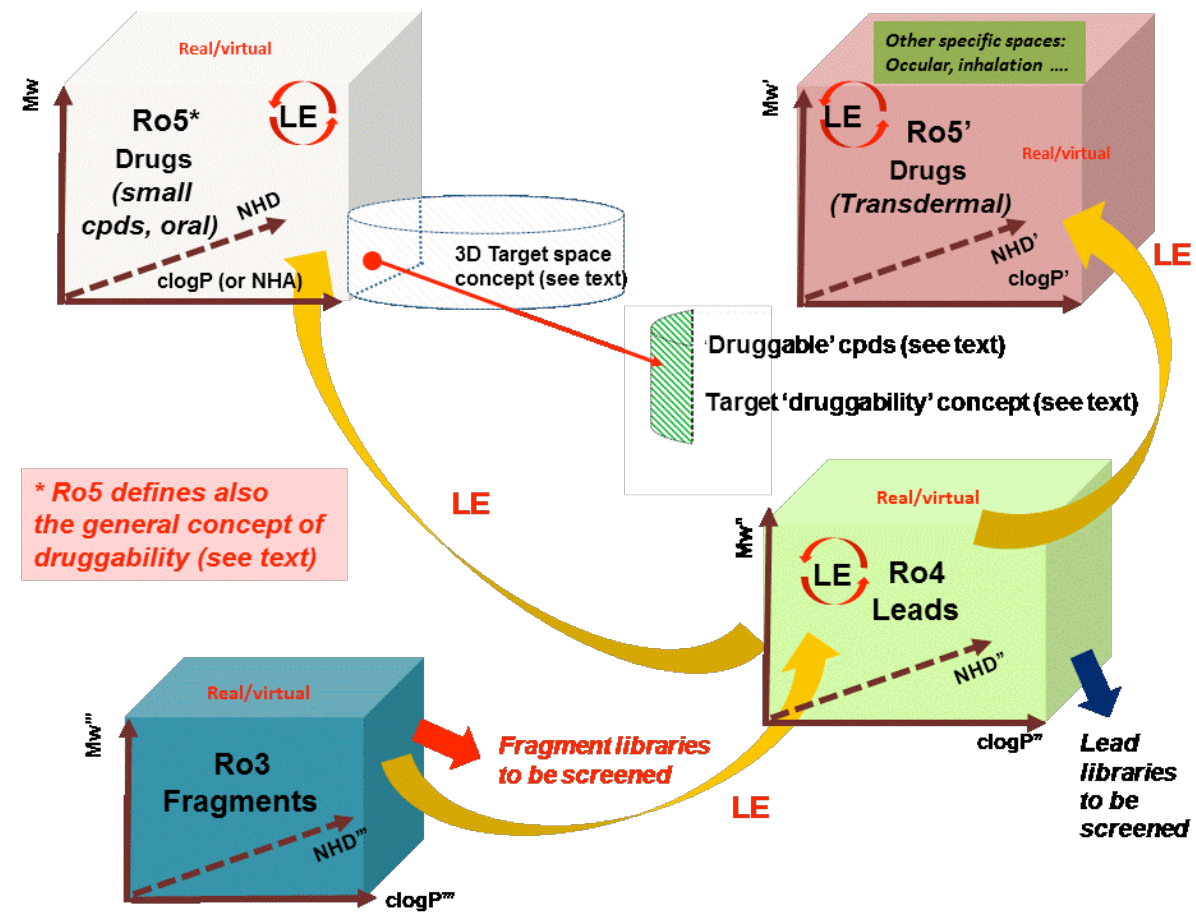

Figure 9. Broad vision for new hits, leads and drug optimization process using the space concept strategy and metrics (LE and Ro5, vide supra).

File: Image (GIF) 


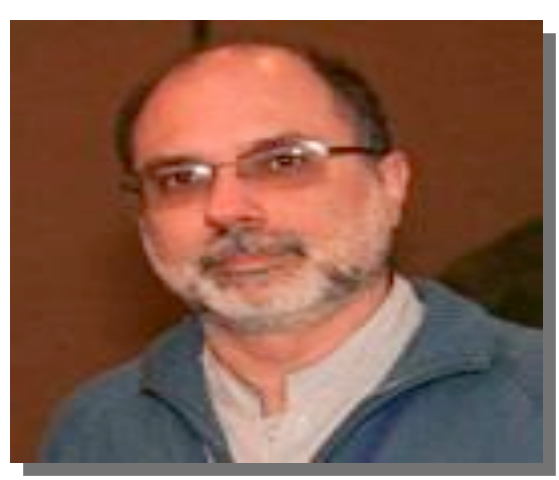




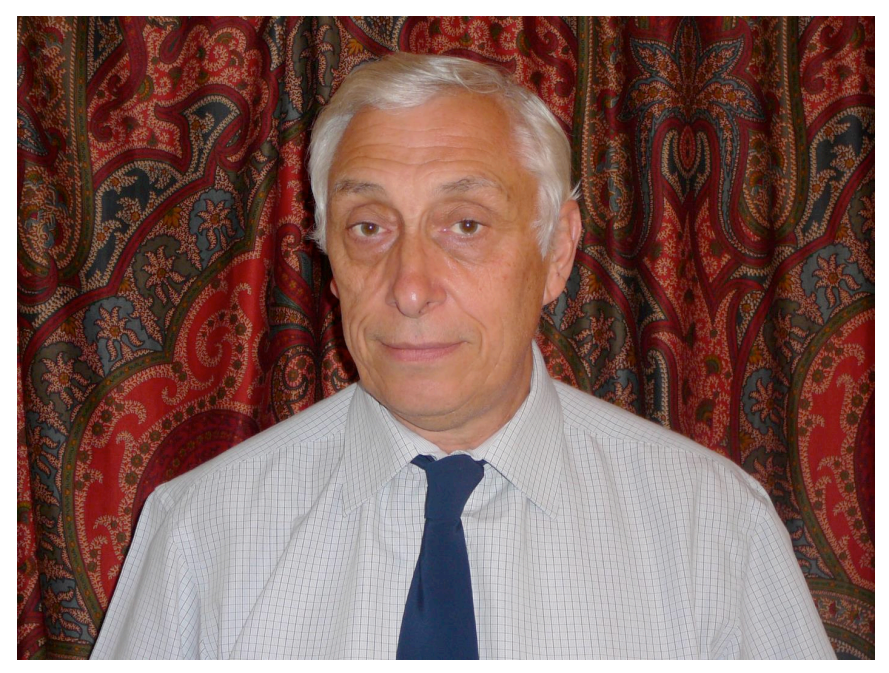




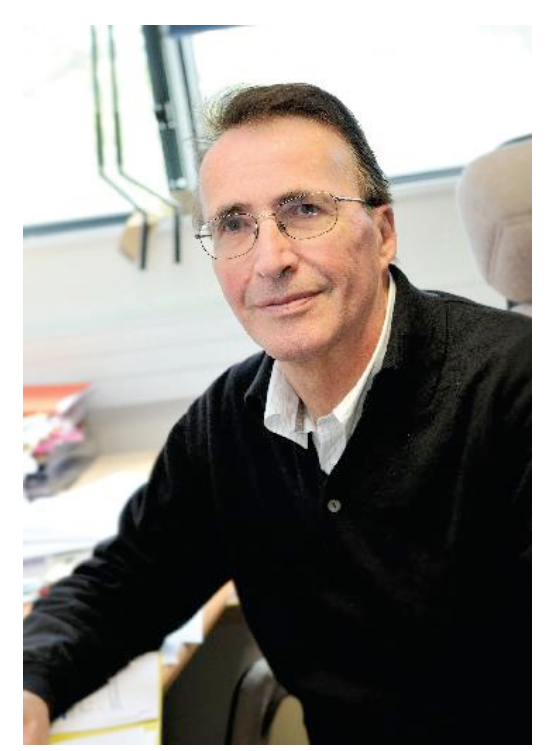

Jean-Pierre Majoral is Emeritus Director of Research at the CNRS in Toulouse. His research interest is focused on the design and the properties of macromolecules such as phosphorus dendrimers and hyperbranched polymers. Main efforts are directed to the use of dendrimers in medicinal chemistry and material sciences. Emphasis is also laid on immobilization of molecular and macromolecular organo- and metal catalysts and their use for fine chemical synthesis. He received a dozen of various prestigious awards from Germany, Poland, Spain, UK and France. He is a member of several Academies of Sciences worldwide and an author of 590 publications and 45 patents. 